\title{
EchoGéo
}

$53 \mid 2020$

Dénominations plurielles. Quand les noms de lieux se concurrencent

\section{Les noms de tranchées et de boyaux de la Grande Guerre en Champagne}

Apport des Plans directeurs des Groupes de Canevas de Tirs des Armées

\section{Alain Devos, Pierre Taborelli et Robin Perarnau}

\section{OpenEdition \\ Journals}

\section{Édition électronique}

URL : https://journals.openedition.org/echogeo/19927

DOI : $10.4000 /$ echogeo. 19927

ISSN : 1963-1197

\section{Éditeur}

Pôle de recherche pour l'organisation et la diffusion de l'information géographique (CNRS UMR 8586)

\section{Référence électronique}

Alain Devos, Pierre Taborelli et Robin Perarnau, «Les noms de tranchées et de boyaux de la Grande Guerre en Champagne », EchoGéo [En ligne], 53 | 2020, mis en ligne le 30 septembre 2020, consulté le 10 août 2021. URL : http://journals.openedition.org/echogeo/19927 ; DOI : https://doi.org/10.4000/ echogeo.19927

Ce document a été généré automatiquement le 10 août 2021.

EchoGéo est mis à disposition selon les termes de la licence Creative Commons Attribution - Pas d'Utilisation Commerciale - Pas de Modification 4.0 International (CC BY-NC-ND) 


\section{Les noms de tranchées et de boyaux de la Grande Guerre en Champagne} Apport des Plans directeurs des Groupes de Canevas de Tirs des Armées Alain Devos, Pierre Taborelli et Robin Perarnau

Les auteurs tiennent à remercier Ophélie Petiot et Marina Clément pour la discrétisation typologique de la base de données toponymique de Champagne, réalisée dans le cadre d'un stage au département de géographie de l'Université de Reims-Champagne-Ardenne. Ils remercient également la région Grand-Est pour le soutien financier du programme IMPACT 14-18, ainsi que les rapporteurs de la revue pour leurs conseils avisés.

\section{Introduction}

1 La Première Guerre mondiale, essentiellement étudiée par les historiens, a lourdement marqué les sociétés par ses héritages géopolitiques, socio-économiques et culturels. Depuis une vingtaine d'années, l'apport des géosciences (Hesse, 2014) et de l'archéologie (Desfossés et al., 2008 ; Brénot et al., 2017) soulève de nouveaux enjeux environnementaux (Masson-Loodts, 2014) dont l'étude constitue encore une niche scientifique, surtout dynamisée par les anglo-saxons (Hupy, 2006 ; Stichelbaut, 2011 ; Gheyle et al., 2016). L'empreinte du conflit est particulièrement marquée dans la zone de front où les héritages sont diversifiés et nombreux, d'ordre pédologique avec les pédoturbations et la bombturbation (Hupy et Schaetzl, 2006), géochimique (Cancès et al., 2018), hydrogéologique (Hubé, 2018 ; Cao et al., 2019), biogéographique (Amat, 2015) et biologique avec les polémochores (Parent, 1991 ; Vernier, 2006 ; Wearn et al., 2017). Les impacts morphologiques avec les polémoformes (Amat, 1987) et plus particulièrement les «fossés " englobant les tranchées et les boyaux des réseaux de défense, sont également étudiés (De Matos-Machado et al., 2016; Devos et al., 2017; 2018a). Ces derniers partiellement ou totalement comblés, induisent des risques de sécurité civile, d'ordre géotechnique (Devos et al., 2019a), pyrotechnique et sanitaire (Hubé, 2016) auxquels sont confrontés les collectivités territoriales mais constituent également un formidable potentiel de valorisation patrimoniale et territoriale. 
2 La toponymie utilisée comme outil de patrimonialisation participe au devoir de mémoire et aux héritages culturels de la Grande Guerre. Les noms de lieux des champs de bataille sont aujourd'hui ancrés dans la mémoire collective, l'historiographie et le territoire. Ils sont pratiqués comme des lieux funéraires et mémoriels de la Grande Guerre. Pourtant, les noms de tranchées et de boyaux restent peu étudiés dans le cadre de la Grande Guerre. L'objet de cette contribution est de les caractériser, de comprendre leur organisation spatiale et leur paternité.

\section{État de l'art}

3 Les noms de tranchées font l'objet de rares ouvrages anglo-saxons (Chasseaud, 2017). Les articles scientifiques, y compris dans les revues d'onomastiques sont quasiinexistants. Leur étude souffre du manque de corpus toponymiques exhaustifs à la différence des toponymes $\mathrm{du}$ cadastre disponibles dans les dictionnaires topographiques départementaux (Longnon, 1891 ; Tamine, 2012). Dans les écrits des combattants, ils sont mentionnés de manière aléatoire sans localisation, sans inventaire et sans informations sur leur organisation spatiale. Les textes traduisent généralement un espace perçu labyrinthique, sans compréhension ni visibilité géographique des noms de lieux. Dans ce dédale des réseaux de défense, la signalétique de tranchée est indispensable pour se repérer. Les communications assurées par des chaines de «coureurs» ou agents de liaison sont difficiles voire aléatoires. L'utilisation de panneaux indicateurs est confirmée par l'approche archéologique (Desfossés et al., 2008) et la consultation des photographies des combattants et des services photographiques des Armées. Cependant, les noms des tranchées restent peu lisibles sur ces photos, disponibles sur les sites internet de la Contemporaine (Collection Valois), de l'ECPAD, de la BNF (Gallica), de l'Imperial War Museum (IWM) et dans les collections privées. De plus, le mobilier archéologique (panneau indicateur) portant un nom de tranchée est rare (Chasseaud, 2017).

4 Les photographies aériennes prises pendant le conflit sont à l'inverse nombreuses. Elles constituent une importante base de données spatiales, dont l'analyse par l'intermédiaire d'un Système d'Information Géographique (SIG) permet d'appréhender l'organisation spatiale des réseaux de défense (Stichelbaut, 2011) et autres structures archéologiques. Elles sont utilisées dans le cadre de la construction des Plans directeurs (Combaud et al., 2016), en collaboration avec le Service de Recherche de Renseignements par Observation Terrestre (SROT), les Sections Topographiques de Corps d'Armée (STCA) et de Division (STDI) et les Sections de Repérage par le Son (SRS). Cependant, les noms de tranchées ne sont reportés sur les levés des cartes que lors de la photo-interprétation et les photographies aériennes ne portent que très rarement d'indications toponymiques.

5 C'est dans la cartographie militaire que les noms de tranchées s'expriment géographiquement. Les manuels d'instructions militaires (GQGA, 1916; 1917) portant sur les Plans directeurs, les cartes et plans spéciaux apportent d'importantes informations sur la désignation des lieux. L'expérience de la guerre montre que pour améliorer l'aspect opérationnel des cartes, la multiplication à l'extrême des noms et désignations s'avère indispensable. Chaque élément des réseaux de défense reçoit des noms ou numéros dans les unités en évitant les noms trop généraux ou génériques tels 
que "parallèle avancée », "nouvelle », " de résistance » ou «boyau central», qui peuvent s'appliquer à toute une région.

6 Les Plans directeurs des Groupes de Canevas de Tirs des Armées (GCTA) sont des cartes à statut secret, réalisées aux 1/20 000,1/10 000 et 1/5000 sur l'ensemble de la zone de front ouest. Elles sont l'équivalent français des Trench maps du Commonwealth et des Stellungskarten allemandes et sont archivées en France au Service Historique de la Défense (SHD), à l'Institut National de l'Information Géographique et Forestière (IGN), dans les services départementaux (archives, bibliothèques, musées) et au sein de collections privées. Des extraits sont utilisés dans les Journaux des Marches et Opérations (JMO) des unités régimentaires engagées dans la Grande Guerre, et consultables sur le site "Mémoires des hommes" du ministère des Armées. Au Royaume-Uni, les cartes sont numérisées et disponibles à la National Library of Scotland et à la McMaster University mais ne concernent que la zone de front occupée par les troupes du Commonwealth (Flandres, Somme). En Allemagne, les Stellungskarten sont disponibles auprès des Archives fédérales, en particulier le Bundesarchiv-Abteilung Militärarchiv de Friburg-en-Brisgau, les Bayerisches Hauptstaatsarchiv de Munich et les Hauptstaatsarchiv de Dresde.

$7 \quad$ Les Plans directeurs représentent l'ensemble des réseaux de défense ennemis en bleu ( Plans directeurs ordinaires ») et alliés en rouge («Plans directeurs avec organisation alliée »). Ils portent des noms de tranchées et de boyaux de même couleur que les réseaux, sur un fond planimétrique en noir sur lequel figure la microtoponymie synthétisée du cadastre (illustration 1). Le relief est représenté en courbes de niveau de couleur bistre, sans ombrage avec un nouveau nivellement. À ce titre, ils constituent un fond toponymique non seulement imposant mais également localisé, avec plus de 20000 noms de tranchées dans la zone de front occupée par le Commonwealth (Chasseaud, 2017). 
Illustration 1 - Extrait du Plan directeur de Reims au 1/20 000 du 15/07/1918, secteur de SaintLéonard

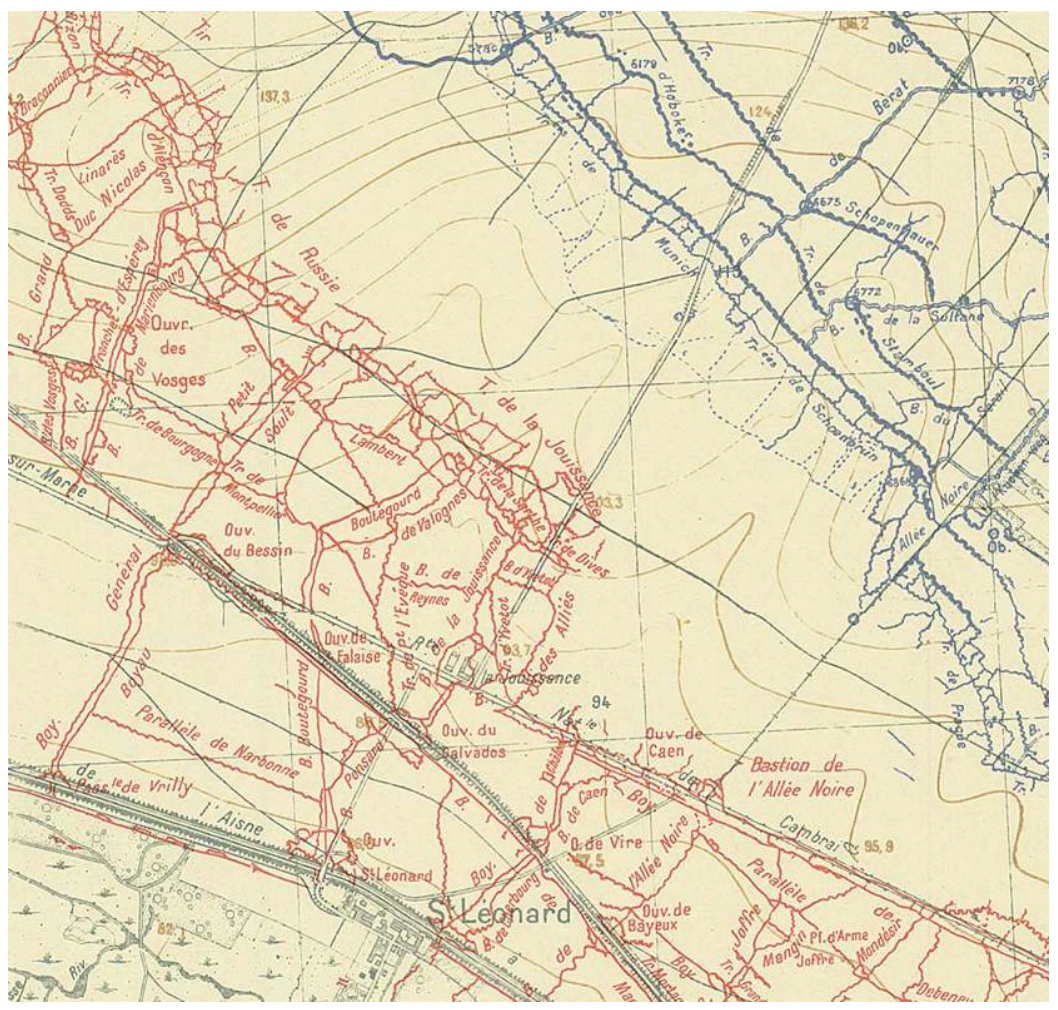

Coll. Personnelle.

\section{Méthodologie}

Dans le cadre du programme de recherches IMPACT 14-18 financé par la Région GrandEst (2015-2018) et porté par l'équipe du GEGENAA de l'Université de Reims ChampagneArdenne, la Champagne traversée par la ligne de front sur $115 \mathrm{~km}$ constitue une zone atelier pour réaliser un diagnostic environnemental post-conflit. Une étude complémentaire à ce programme a été menée sur les noms de tranchées dans la zoneatelier, comprise entre l'Aisne médiane à Berry-au-Bac à l'ouest, et l'Aisne supérieure en Argonne à l'est. Ce secteur correspondant à $15 \%$ du linéaire du front ouest est représentatif de l'ensemble du dispositif de défense de la guerre de position et ne connaît pas de grand retrait stratégique ni mouvance importante du front comme dans la Somme (retrait Alberich) qui double la densité de tranchées et de boyaux.

Dix Plans directeurs communiqués par la DRAC Grand Est (Direction Régionale des Affaires Culturelles), avec organisations alliées au 1/20000 de 1918, numérisés et géoréférencés couvrent la zone atelier. Une banque de données spatiales est constituée dans le système de projection Lambert 93 (RGF 93) par digitalisation manuelle et représentée par des figurés ponctuels (observatoires, ponts et batteries), linéaires (tranchées et boyaux), et enfin polygonaux (centres et réduits de défense). Les noms de tranchées allemandes et françaises sont géolocalisés et référencés dans la base. Ils désignent chacun un segment des réseaux de défense qui sont étudiés dans le cadre d'une cartographie sous SIG impliquant l'utilisation d'indices morphométriques (longueurs, densités linéaires et surfaciques, largeur du No man's land). 
10 Les noms de tranchées font l'objet d'une typologie en neuf classes ou groupes, réalisée manuellement : temps, toponymes (lieux/espace), ergonymes (objets), anthroponymes (personnes), zoonymes (animaux), phytonymes (végétaux), phénonymes (phénomènes non-verbaux), chiffres/lettres et autres. Chaque groupe se décline en 24 sous-groupes, divisés en 19 particularités. Le paramètre temporel est divisé en périodes historiques, les toponymes comme noms de lieux préexistants, hydronymes ou oronymes. Les résultats sont valorisés graphiquement et par une cartographie typologique sous SIG permettant de représenter l'organisation spatiale des 9 classes.

\section{Résultats}

11 La cartographie des réseaux de défense réalisée sous SIG sur les $115 \mathrm{~km}$ de front (illustration 2), dévoile un réseau épais, complexe et structuré. Il se compose de 47212 segments sur $13059 \mathrm{~km}$ de linéaire total dont $7014 \mathrm{~km}$ de tranchées et $6045 \mathrm{~km}$ de boyaux déterminant une densité moyenne de 3,63 segments $/ \mathrm{km}$ linéaire ou un segment élémentaire de $263 \mathrm{~m}$ (tableau 1).

\section{Illustration 2 - Carte des réseaux de défense de 1918 en Champagne}

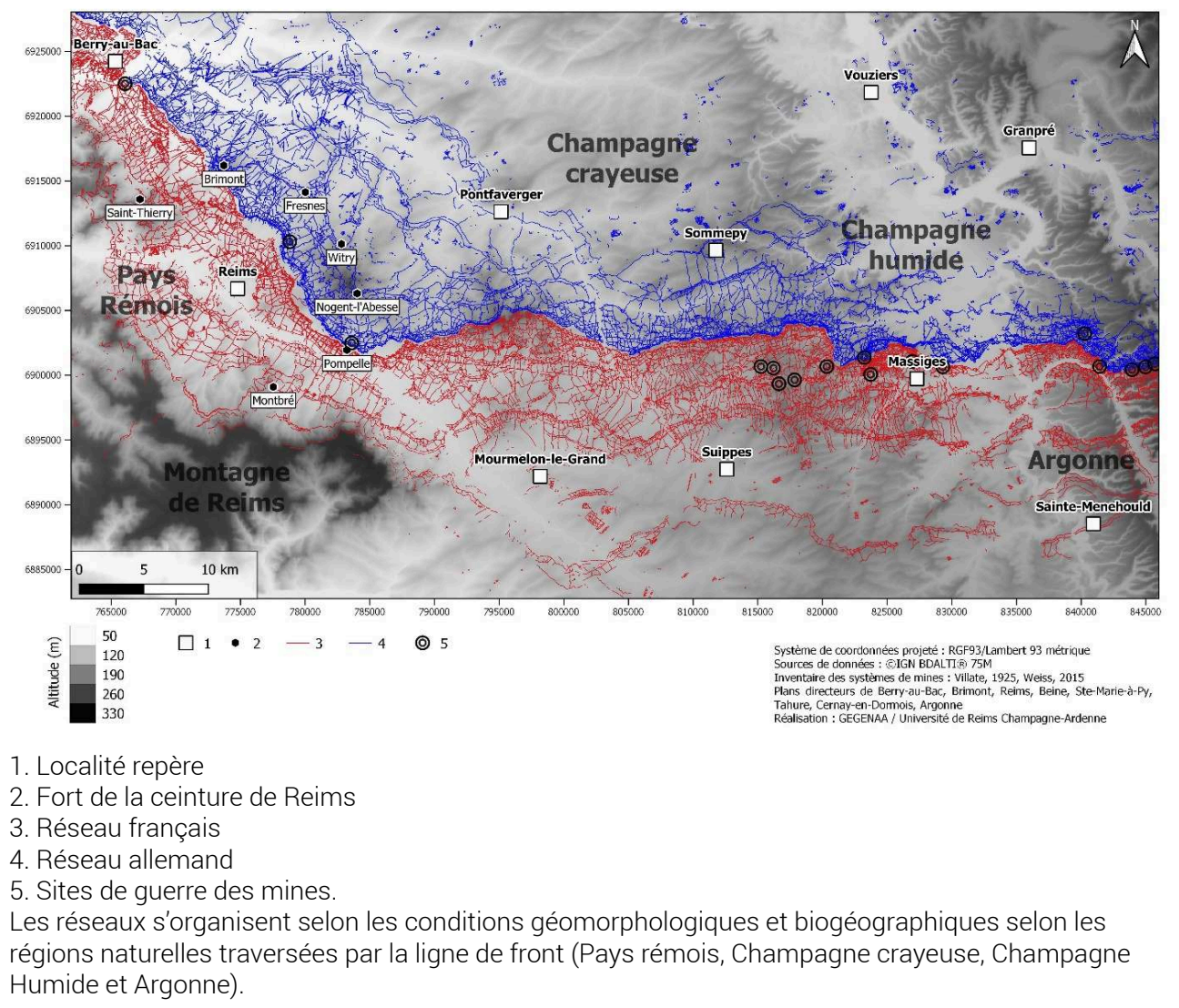


Tableau 1 - Caractéristiques morphométriques et toponymiques des réseaux de défense de Champagne

\begin{tabular}{|l|c|}
\hline \multicolumn{1}{|c|}{ Paramètre } & Valeur \\
\hline Longueur de la ligne de front $(\mathrm{km})$ & 115 \\
\hline Nombre de segments & 47212 \\
\hline Linéaire total $(\mathrm{km})$ & 13059 \\
\hline Linéaire de tranchées $(\mathrm{km})$ & 7014 \\
\hline Linéaire de boyaux $(\mathrm{km})$ & 6045 \\
\hline Ratio linéaire de tranchées/boyaux & 1.16 \\
\hline Densité linéaire $\left(\mathrm{km} / \mathrm{km} \mathrm{de} \mathrm{ligne} \mathrm{de} \mathrm{front)}^{2}\right.$ & 113 \\
\hline Densité surfacique $\left(\mathrm{km} / \mathrm{km}^{2}\right)$ & 4.87 \\
\hline Nombre de noms de tranchées et de boyaux & 2683 \\
\hline Densité toponymique linéaire $($ nombre $/ \mathrm{km})$ & 0.21 \\
\hline Densité toponymique $\left(\right.$ nombre $\left./ \mathrm{km}^{2}\right)$ & 2.34 \\
\hline Longueur de segment nominatif $(\mathrm{km})$ & 4.87 \\
\hline
\end{tabular}

12 Ces chiffres se traduisent par une densité surfacique de réseau de $4,87 \mathrm{~km} / \mathrm{km}^{2}$, et une densité linéaire de $113 \mathrm{~km} / \mathrm{km}$ de front. Les réseaux se développent de part et d'autre du No man's land en trois à quatre positions (ou rideaux) structurées chacune par une à 14 lignes de tranchées. Le dispositif s'étend sur une surface de $6281 \mathrm{~km}^{2}$, avec une épaisseur comprise entre 20 et $60 \mathrm{~km}$.

Dans cette zone de front, 2683 noms de tranchées et de boyaux sont recensés, ce qui détermine une densité toponymique surfacique de $2,34 / \mathrm{km}^{2}$, inférieure à la densité moyenne française de 3,1 toponymes $/ \mathrm{km}^{2}$ de la BDNYME ${ }^{\circledast}$ de l'IGN comprenant 1700000 toponymes pour l'hexagone (Tamine, 2012). Cependant, ce paramètre est biaisé par une répartition spatiale très hétérogène des tranchées et des boyaux. L'organisation spatiale de la densité toponymique paraphrase celle des réseaux de défense. En effet, la densité intègre d'importantes surfaces, correspondant aux « intervalles » entre les positions. La lecture des Plans directeurs montre que cette densité est bien plus élevée (près de 10 toponymes $/ \mathrm{km}^{2}$ ), dans les positions de défense que dans les intervalles traversés par de rares boyaux (moins de deux toponymes).

Cette densité diminue corrélativement à l'éloignement de la ligne de front. Dans la région de Reims, les premières positions sont effectivement bien plus denses $(13 \mathrm{~km} /$ $\mathrm{km}^{2}$ ) que les quatrièmes positions $\left(4 \mathrm{~km} / \mathrm{km}^{2}\right)$ situées en Montagne de Reims (Devos et al., 2015). En Argonne, le premier rideau allemand hypertrophié et comportant 14 lignes de tranchées concentre donc la majeure partie des toponymes (Taborelli et al., 2016).

15 La densité toponymique linéaire de $0,21 / \mathrm{km}$ linéaire se traduit par une longueur moyenne de segments de tranchée ou de boyau, désigné par un nom, de 4,89 km. Ces chiffres moyens cachent également d'importantes disparités spatiales inhérentes à l'organisation des réseaux de défense. Cette densité diminue corrélativement à l'éloignement de la ligne de front. Elle dépend du cadre géographique et des paramètres militaires balistiques (portée de tir) et tactiques (Vilatte, 1925). La multiplication des petits postes avancés et observatoires, en première position détermine un chevelu de boyaux courts, désignés par des noms spécifiques. 
Tableau 2 - Typologie des 2683 noms de tranchées et de boyaux de Champagne en 1918

\begin{tabular}{|c|c|c|c|c|}
\hline Typologie & Groupe & Effectif & $\%$ & Effectif par sous-groupe \\
\hline Temps & $\mathbf{T}$ & 33 & 1.2 & $\begin{array}{l}0 \text { Période historique, } 0 \text { préhistoire, } 30 \text { faits } \\
\text { historiques, } 3 \text { dates }\end{array}$ \\
\hline Lieux & $\mathbf{L}$ & 1154 & 43 & $\begin{array}{l}12 \text { toponymes préexistants, } 76 \text { hydronymes, } 14 \\
\text { géonymes, } 64 \text { oronymes, } 50 \text { biomes, } 213 \text { pays et } \\
\text { territoires, } 708 \text { villes et villages, } 17 \text { points cardinaux }\end{array}$ \\
\hline Ergonymes & E & 255 & 9,5 & $\begin{array}{c}12 \text { marques et entreprises, } 69 \text { établissements } \\
\text { civils, } 11 \text { religieux, } 72 \text { militaires, } 7 \text { culturels, } 27 \\
\text { objets militaires, } 51 \text { du quotidien, } 3 \text { mots } \\
\text { d'argot, } 3 \text { couleurs }\end{array}$ \\
\hline Anthroponymes & A & 879 & 32,8 & $\begin{array}{l}671 \text { patronymes, } 20 \text { pseudonymes, } 36 \\
\text { ethnonymes, } 22 \text { ethnologie, } 51 \text { titres, } 22 \\
\text { métiers, } 49 \text { grades et affiliations militaires, } 8 \\
\text { caractères physique }\end{array}$ \\
\hline Règne animal & $\mathbf{R}$ & 137 & 5,1 & $\begin{array}{l}59 \text { mammifères, } 6 \text { reptiles, } 16 \text { poissons et } \\
\text { amphibiens, } 46 \text { oiseaux, } 2 \text { invertébrés, } 8 \\
\text { insectes }\end{array}$ \\
\hline Règne végétal & $\mathbf{V}$ & 38 & 1,4 & 19 arbres, 8 arbustes, 6 herbes, 5 légumes \\
\hline Phénonymes & $\mathbf{P}$ & 19 & 0,7 & $\begin{array}{c}7 \text { cosmologie, } 3 \text { géologie, } 8 \text { médecine, } 1 \text { santé } \\
\text { et sport }\end{array}$ \\
\hline Chiffres et lettres & C & 125 & 4,7 & \\
\hline Divers/non définis & $\mathbf{N}$ & 43 & 1,6 & \\
\hline & & 2683 & & \\
\hline
\end{tabular}

Illustration 3 - Répartition des classes toponymiques des noms de tranchées et de boyaux de Champagne en 1918

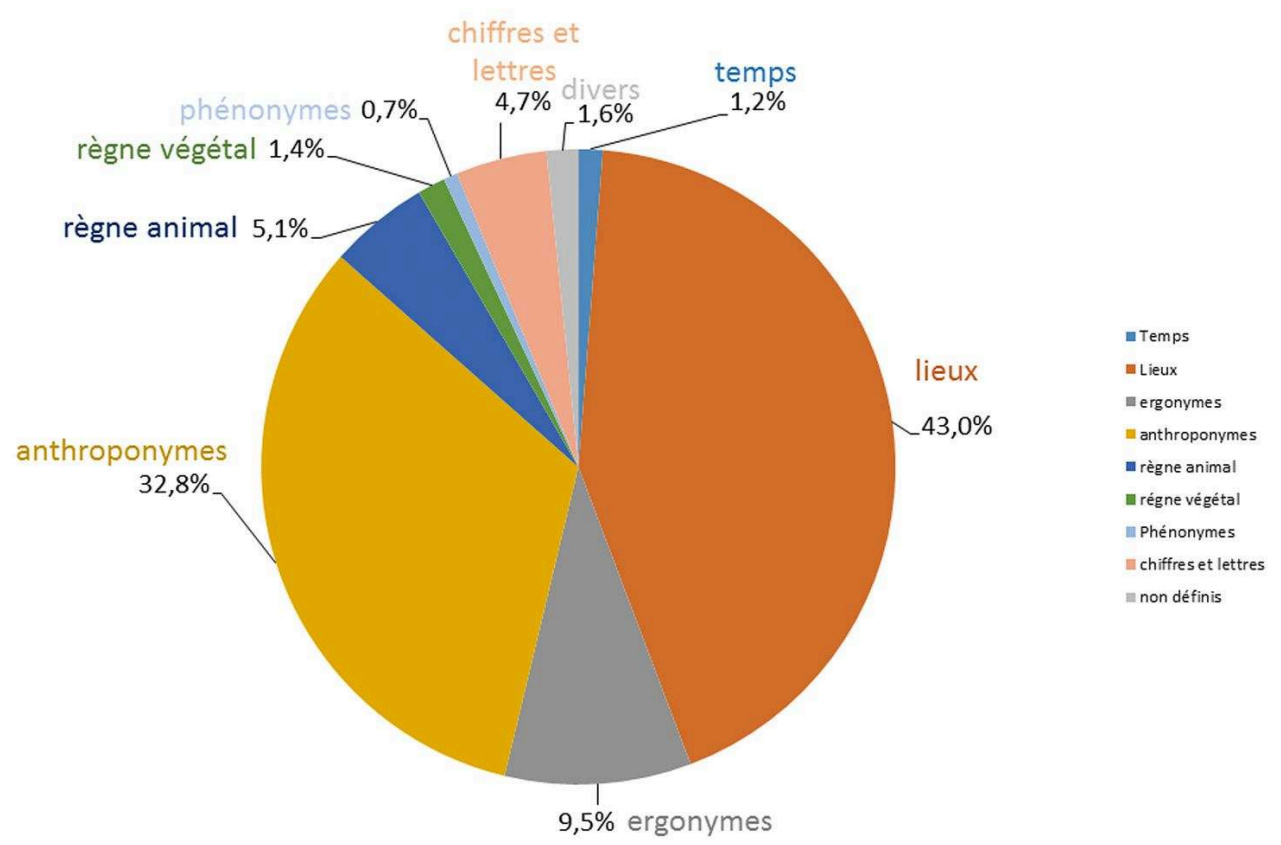

\section{Les toponymes en tête des noms les plus utilisés}

La désignation des tranchées et des boyaux est marquée par une grande diversité lexicale mise en évidence par l'analyse typologique (tableau 1, illustration 3). La distribution est bimodale car les noms de lieux (43\%) ainsi que les anthroponymes (33\%) sont privilégiés, constituant $76 \%$ de l'effectif total (illustration 4 ). Viennent ensuite les ergonymes (9,5\%), les zoonymes et les phytonymes (6,5\%), les chiffres et les 
lettres $(4,7 \%)$, les noms de lieux relatifs au temps ou à l'histoire $(1,2 \%)$, et enfin les phénonymes $(0,7 \%)$.

Les toponymes sont surtout représentés par des noms de villes, villages ou quartiers (708 soit $26 \%$ ) principalement internationaux (54\%), nationaux ( $28 \%$ ) ou régionaux $(18 \%)$. Cette répartition s'explique par la dénomination des tranchées ennemies par des noms de villes germaniques comme les «Tranchées de Hambourg, de Munich, de Berlin, de Postdam » conformément aux recommandations des manuels d'instructions militaires (GQGA, 1916; 1917). La mondialisation toponymique s'explique également par la présence de troupes étrangères alliées tels les Russes dans le secteur de la Pompelle près de Reims ("Tranchée de Pétrograd») ou les Italiens en Argonne et à l'ouest de Reims (Tableau 2).

Illustration 4 - Distribution des 2683 noms de tranchées et de boyaux en Champagne par sousgroupes toponymiques

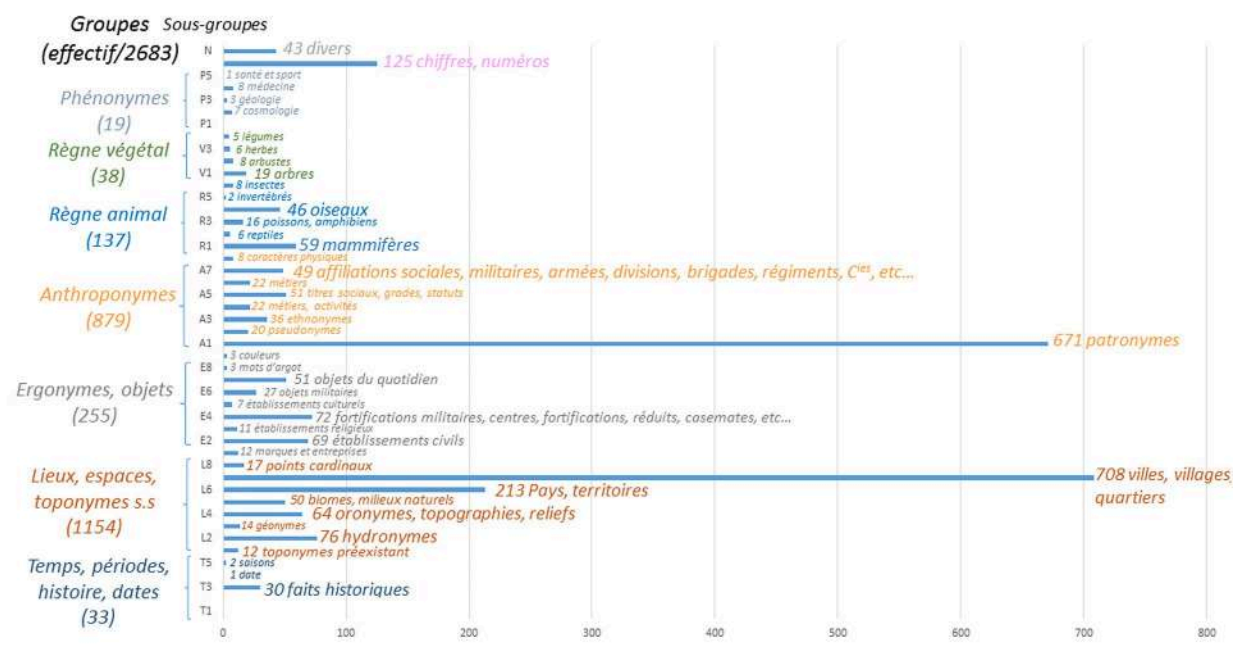

213 toponymes (soit $10 \%$ de l'effectif total) désignent des pays et territoires étrangers "Tranchée de Russie ", ou nationaux avec des régions françaises à l'image des boyaux « d'Algérie, de Picardie, de Normandie ». Citons également les quelques hydronymes au nombre de 76 ( $3 \%$ de l'effectif total) désignant des tranchées ou boyaux : « parallèles de l'Amazone, du Gange, de l'Euphrate, de l'Ontario, ou du Rhône » dans le secteur de Saint Hilaire le Grand. Cette faible proportion d'hydronymes, peut s'expliquer par une densité de drainage particulièrement faible en Champagne sèche, inhérent à la perméabilité de la craie affleurante. Les noms de reliefs sont également usités de la même ampleur que les précédents (64 mentions). Enfin, les noms locaux empruntés au fond toponymique local ou régional («boyaux de Verzy et de Verzenay») traversent logiquement les villages éponymes mais sont très peu utilisés pour désigner les noms des réseaux de défense (12 mentions).

\section{Les anthroponymes comme second mode de la distribution}

19 Avec 879 références (33\%), les anthroponymes et plus particulièrement les 671 patronymes, constituent le second mode de la distribution. Ils sont essentiellement 
représentés par des noms de militaires (63\% des patronymes) et de personnages historiques (22\%).

Aux "Charles Quint ", «François Joseph», «Ferdinand 1 $1^{\mathrm{er}}$ ", «Bismark», «Von Kluck», ou "Kaiser» des réseaux allemands, s'opposent les "Pétain», "Foch», «Joffre ", « Mangin », « Nivelle ", «Gallieni », «Franchet-d'Esperey » et «Jeanne d'Arc» pour les tranchées et boyaux français. Cette écrasante majorité de dénominations militaires est à rattacher également à l'organisation cellulaire des réseaux autour de centres ou réduits de résistance désignés par le nom des officiers, capitaines ou commandants qui les dirigent comme les centres « Lambert ", " Jubault ", « Dufour » et « Clausse » en Champagne sèche.

\section{Une grande diversité de noms peu employés}

Les autres appellations ( $28 \%$ de l'effectif total) sont certes diversifiées comme les ergonymes, les chiffres et lettres, les zoonymes, les phytonymes, et les phénonymes mais restent minoritaires car elles représentent respectivement 9,5\%, 4,7\%, 5, 1\%, $1,4 \%$ et $0,7 \%$ des 2683 noms de tranchées (illustration 4 ).

Les ergonymes (9,5\%), sont principalement empruntés au vocabulaire des fortifications, forteresses, réduits, centres, casemates (72) et à des objets militaires (27) du champ de bataille comme les types d'avions allemands (tranchées "de l'Aviatik ", "du Taube», "de l'Albatros», "du Fokker», ou les boyaux «du Gotha » et "des Avions». Les armes des tranchées ne sont pas oubliées comme les boyaux «de la Torpille », « du Minen » ou « de la Mitrailleuse ».

Les numéros et les lettres ne sont utilisés qu'à titre exceptionnel (125 mentions soit $4,7 \%$ de l'effectif total), conformément aux instructions des manuels militaires qui réservent les chiffres aux coordonnées géographiques des nœuds de tranchées et des batteries. La numérotation à trois chiffres reste conventionnelle et doit éviter toute répétition en permettant de rajouter un numéro sans modifier l'état initial et en respectant une logique à la fois mathématique et spatiale, par position de défense (par exemple des numéros commençant par « 1 » en première position, par « 2 » en seconde puis « 3 » en troisième. Le second chiffre peut correspondre aux coordonnées géographiques, soit de l'abscisse pour un déplacement d'est en ouest, soit de l'ordonnée pour une suite numérique du sud au nord.

24 La désignation par une ou plusieurs lettres précédant un chiffre d'éléments de tranchées ennemis peut être usitée comme les «B. $21 »$, «B. $21^{\text {bis }}$, «B. $22 », ~ « B .22^{\text {bis }}$, «B. $22^{\text {ter }}$ » du Plan directeur de Saint-Souplet du 10 septembre 1918. Cette désignation est surtout utilisée en première position, à partir du parallèle de résistance, pour les petits postes avancés ou «P.P.» dirigés vers le No man's land. Ces derniers sont particulièrement nombreux et donc logiquement désignés par leur sigle précédant un chiffre au sein du fuseau géographique correspondant, comme les «P.p.3Y », «P.p.4Y » dans le fuseau Y ou le «P.p.8Z » du fuseau Z.

Les phytonymes et les zoonymes sont rarement mentionnés avec 19 arbres, 59 mammifères, 46 oiseaux, 16 poissons et amphibiens (6,5\% de l'effectif total). Ils taxent les tranchées et boyaux de noms fantasques comme les tranchées "des Sardines ", " du Silure », " de la Salamandre ", " de la Sangsue », " de la Chouette ", « de la Caille » ou « du Castor ». 
Les noms particuliers, sobriquets, et injures sont rares (43 mentions sur 2683 ). Ils désignent surtout le dispositif ennemi pour le dénigrer ou l'humilier comme le «boyau du Vampire", les tranchées "des Satyres", "des Démons", «des Huns", "des Boches ", «des Vandales", "des Pirates ", «des Eunuques", "des Têtes Carrées", "des Homosexuels » ou "tranchée Fantômas » et plus rarement des tranchées alliées comme les tranchées «du Sagouin » et "du Sapajou ». On ne retrouve donc que très localement, dans la toponymie du champ de bataille, la légitimation culturelle de la guerre de la civilisation contre la Kultur ou la barbarie allemande véhiculée dans l'iconographie propagandiste (Danchin, 2011).

Enfin, les noms de lieux relatifs au temps ou à l'histoire (1,2\%), et enfin les phénonymes $(0,7 \%)$ restent anecdotiques. Ils auraient pu être versés dans la classe des toponymes urbains mais correspondent à des batailles napoléoniennes comme les boyaux « d'Iéna », « Marengo », « d'Arcole », « Friedland » ou la « Tranchée Wagram ».

\section{Discussion}

L'inventaire des noms de tranchée à partir des Plans directeurs de 1918, montre que l'appropriation du champ de bataille par les militaires se manifeste par une grande diversité et un foisonnement d'appellations. La densité toponymique traduit un désir d'appropriation de l'espace durement gagné ou conquis voire à conquérir.

La multiplication des noms conformément aux instructions des manuels militaires, répond au souci de géolocalisation dans un réseau de défense particulièrement dense et épais, formant un véritable labyrinthe où sans signalétique, sans guide et sans cartographie, la perdition ou l'errance sont inévitables. L'inventaire des noms de tranchées soulève de multiples questionnements sur leur répartition spatiale, leur origine, leur permanence dans le temps et dans l'espace selon la dimension scalaire (du $1 / 20000$ au 1/5 000) et sur l'héritage toponymique de la Grande Guerre en Champagne.

\section{Une répartition spatiale révélant une récurrence toponymique à l'échelle locale}

L'approche spatiale sous SIG des types de noms de tranchées fait apparaître les tranchées affublées d'un nom et celles qui en sont dépourvues (illustration 5). Les couleurs dominantes rouges et bleues paraphrasent la distribution bimodale des noms autour des noms de lieux (villes, villages, territoires) et les anthroponymes. Si de prime abord, l'organisation spatiale des classes de noms de tranchées s'avère aléatoire à l'échelle de la Champagne, dans le détail, à l'échelle locale, des segments de tranchées de même classe toponymique semblent ressortir. 
Illustration 5 - Répartition spatiale des groupes toponymiques des tranchées et boyaux en Champagne en 1918

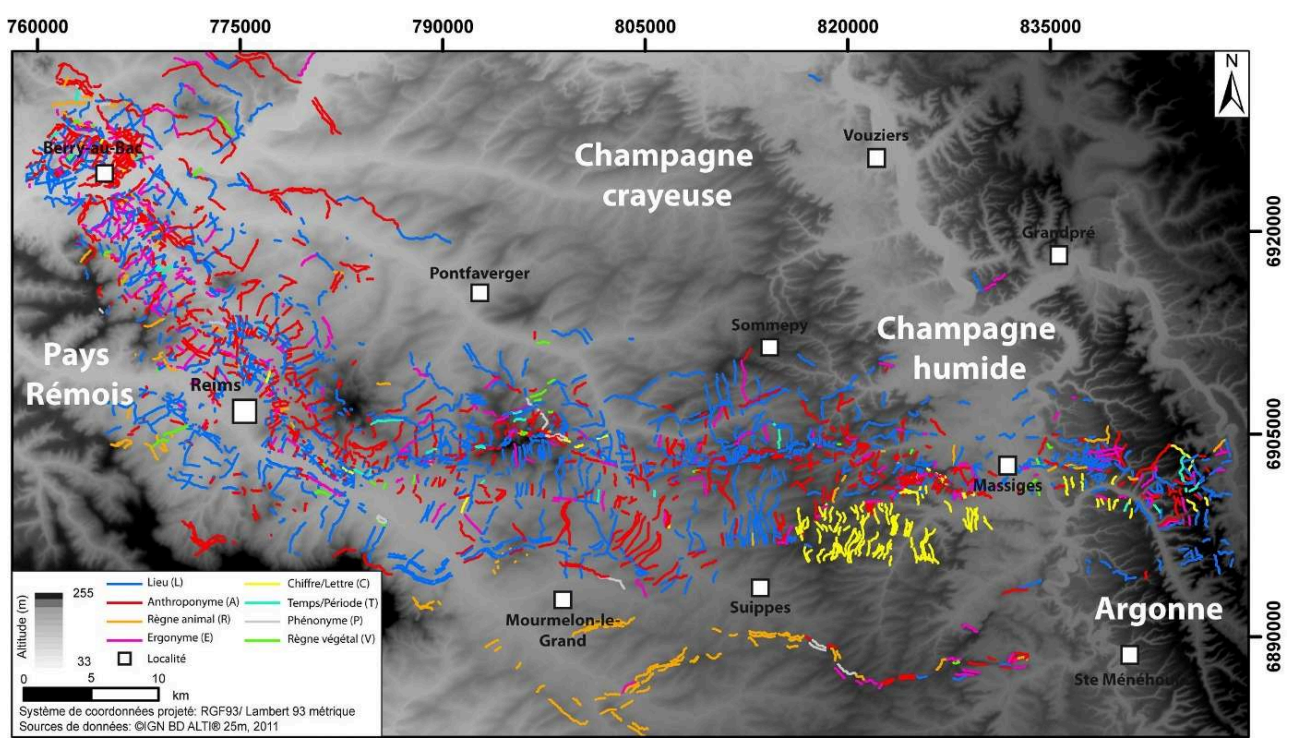

31 La $4{ }^{\text {ème }}$ position française au sud de Mourmelon-le-Grand et de Suippes, est davantage représentée par des noms d'animaux qui après analyse commencent systématiquement par la lettre « $\mathrm{S}$ » comme les tranchées «de la Sardine», «du Sanglier», "du Scorpion », « du Silure ", « de la Souris", " de la Salamandre». Le secteur en $3^{\text {ème }}$ position française entre Massiges et Suippes, se caractérise par des boyaux désignés par des chiffres et des lettres dont la logique obéit au carroyage Lambert zone Nord de guerre (boyaux «B12», «B13», «B14») et dont le second chiffre correspond soit à l'abscisse soit à l'ordonnée.

Près de Saint-Hilaire-le-Grand, des tranchées et parallèles portent le nom d'hydronymes mondiaux ("Gange », «Euphrate », " Tigre », " Ontario »). Au sud du fort de la Pompelle près de Reims, des boyaux portent le nom d'officiers français ( Joffre », « Mangin », « Nivelle », « Pétain », « Foch », « Franchet d'Espérey ", « Petit ») sur un à deux km de front. À l'est du fort de la Pompelle, le nom des tranchées rappelle l'occupation russe du secteur ("Baltique», "Sibérie», "Oural », "Pétrograd», "Crimée », " Caucase », « Lodz » ou "Kovel ») sur un km de front (illustration 6D). Au nord-est de Reims dans le secteur de Saint-Léonard, les noms de tranchées sont tous d'origine normande avec les tranchées « de Dives ", " d'Yvetot », les boyaux " de Vire », «de Cherbourg», les ouvrages "du Calvados», "de Caen», " de Falaise», "de Bayeux » sur un territoire de moins de $2 \mathrm{~km}^{2}$ (illustration 1 ).

Toujours à l'est de Reims, au niveau du quartier Jeanne d'Arc, les boyaux «Jeanne d'Arc, de Rouen, de Domrémy ", l'ouvrage «Jean d'Arc », la "Tranchée d'Orléans » rappellent le trajet mythique de Jeanne d'Arc et constituent un réseau de défense sur $500 \mathrm{~m}$ de ligne de front (illustration 6C).

34 À l'est de Sept-Saulx, un réseau toponymique homogène toujours sur un $\mathrm{km}$ de front fait référence aux États-Unis d'Amérique avec les parallèles de «Chicago », «du Mississipi », " de l'Ohio », " du Niagara », " du président Wilson », " de l'Oncle » (illustration 6B). Enfin, dans le secteur de la ferme de l'Espérance entre Sillery et Beaumont-sur-Vesle, un groupe de tranchées désigne des minéraux comme les parallèles " du Diamant ", " du Saphir », " des Topazes » et "des Améthystes » sur un $\mathrm{km}$ de front (illustration $6 \mathrm{~A}$ ). 
La lecture attentive des Plans directeurs fait donc apparaître une organisation spatiale des classes toponymiques à l'échelle locale. Les références culturelles à des épisodes du roman national français (parcours de Jeanne d'Arc) et à des personnages que l'on veut glorifier parmi les officiers contemporains («Pétain», «Foch») traduisent un certain patriotisme guerrier. En revanche, les références à des territoires («Sibérie», "Chicago » par exemple) font écho à l'origine géographique des soldats (la Russie pour le secteur du fort de la Pompelle par exemple, illustration 6D). Cette homogénéité des classes à l'échelle locale témoigne d'une logique de dénomination particulière.

\section{Illustrations 6 A et $B$ - Exemples de récurrence spatiale et thématique des désignations de} tranchées à l'échelle locale
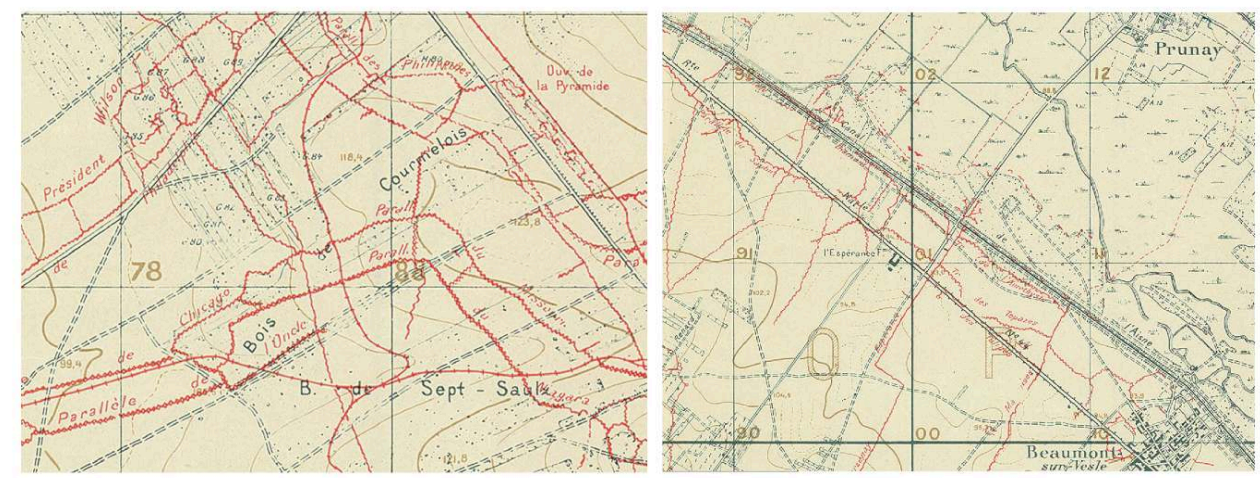

Gauche : A. Les États-Unis d'Amérique - Droite : B. Pierres précieuses et minéraux.

Sources : Extraits de la feuille de Beine du 10 août 1918, coll. Personnelle.

Illustrations $6 \mathrm{C}$ et $\mathrm{D}$ - Exemples de récurrence spatiale et thématique des désignations de tranchées à l'échelle locale
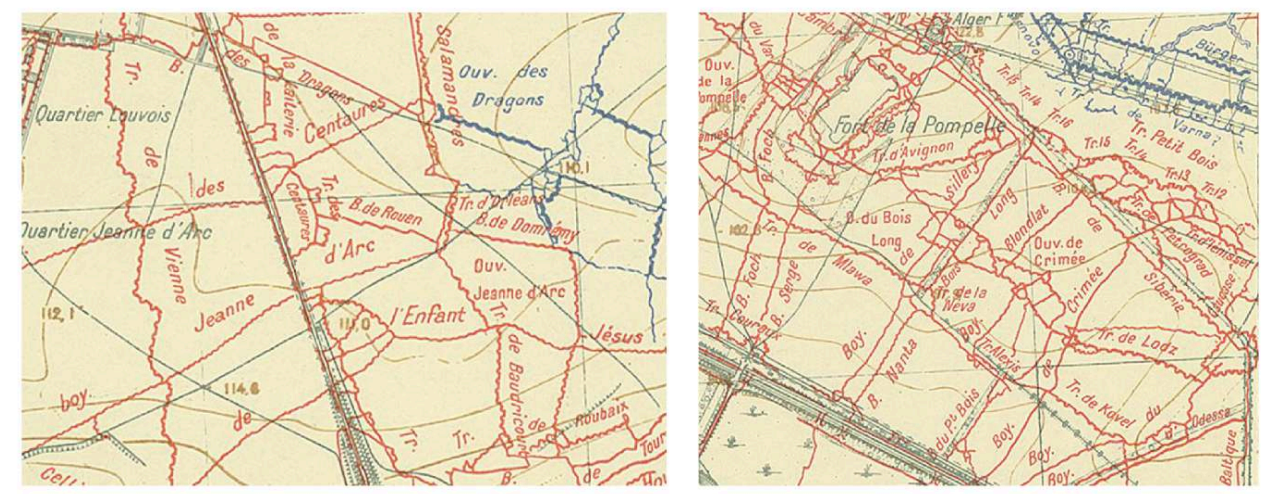

Gauche : C. Trajet de Jeanne d'Arc - Droite : D. La Russie.

Sources : Extraits de la feuille de Reims du 15 juillet 1918, coll. Personnelle.

\section{Une récurrence spatiale et culturelle témoignant d'une origine régimentaire et non divisionnaire.}

L'amplitude fluctuante des toponymes, comprise entre quelques centaines de mètres et $10 \mathrm{~km}$ semble également augmenter corrélativement à l'éloignement du front. Les segments de même classe toponymique sont effectivement plus longs dans les positions de l'arrière (réseau moins labyrinthique) qu'aux abords de la ligne de front. En effet, en 
première position, l'espace élémentaire désigné par un groupe de noms selon un thème, semble par contre bien restreint, réduit à quelques centaines de mètres. La taille de cet espace permettrait ainsi de connaître l'origine des désignations.

L'organisation opérationnelle militaire est perpendiculaire au front et s'inscrit dans un emboitement d'échelles où l'espace est affecté à une unité militaire. Sur les $750 \mathrm{~km}$ de linéaire de front, les alliés alignent des Armées affectées chacune à une «zone d'action ». Chaque Armée s'organise de la manière suivante :

38 - Elle se divise en "Corps d'Armée » sur une « zone d'engagement »;

- Un «Corps d'Armée» affecte un «secteur d'engagement» propre à chaque "division» (les alliés alignent 93 à 119 divisions contrôlant chacune un «secteur d'engagement » compris entre six et huit km de front) ;

- Une «Division" répartie un «sous-secteur d'engagement» pour un ou plusieurs régiments («Brigade »);

- Une « Brigade » affecte un « quartier d'engagement » pour un « bataillon » ou un seul régiment ;

- Une «Compagnie » régimentaire contrôle un « sous quartier d'engagement » dans un point d'appui ou un réduit ;

- Enfin, une «Section» d'une «Compagnie» est responsable d'un «Groupe de combat".

39 L'espace élémentaire toponymique proscrit toute logique divisionnaire ou de Corps d'armée puisque l'espace moyen tenu par une division est plurikilométrique (Devos et al., 2018b). Il correspond davantage à un secteur tenu par un régiment (moins d'un kilomètre de front), voire par une compagnie ( $200 \mathrm{~m}$ de front). La désignation toponymique des tranchées semble être l'apanage des troupes occupant le terrain. À ce titre, celles-ci désignent les tranchées et boyaux qu'elles occupent par la marque de leur histoire régimentaire, de leur ville de garnison, de leur culture militaire ou du secteur fortifié. À noter que la grande diversité culturelle, la faible représentativité des noms d'argot de tranchée (Sainéan, 1915) ainsi que l'espace élémentaire toponymique proche du «quartier » feraient référence à une désignation faite par des officiers supérieurs voire subalternes.

\section{Une paternité régie selon des logiques militaires, géographiques et historiques}

40 Conformément aux instructions des manuels militaires, les tranchées creusées reçoivent les noms et numéros usités dans les unités qui s'approprient le terrain (GQGA, 1916 ; 1917). Cette désignation aléatoire présente néanmoins des logiques militaires dans la paternité des noms de tranchées. La désignation des centres de résistance abritant les postes de commandement, fait souvent référence au premier chef de bataillon qui les dirige, ce qui entretient la forte densité patronymique. Les tranchées portent souvent le nom de militaires morts, pendant les opérations de creusement ou qui commandaient à leur exécution. C'est le cas du Commandant Jean-Marie Cano du $247^{\text {ème }}$ Régiment d'Infanterie, qui meurt le 25 août 1915 lors de l'aménagement d'une tranchée au Bois des Bouleaux près de Souain en Champagne sèche (ministère des Armées, 1915). Cette appropriation du terrain par la toponymie renforce la valeur identitaire et l'esprit de corps du groupe de combat. 
41 Les noms de tranchées font également référence au dispositif de défense à l'image des centres « des écoutes » et « des guetteurs » situés en première position, ou le " réduit intermédiaire» entre deux positions. Les noms des tranchées ennemies des Stellungskarten sont, reportés si possible sur les Plans directeurs, d'où l'importance des « coups de mains » visant à capturer des prisonniers et à disposer de leurs cartes.

\section{La permanence scalaire et temporelle des noms de tranchée}

42 Conformément aux instructions des manuels militaires, il est de règle que tous les noms de tranchées portés sur les Plans directeurs au 1/20 000 figurent sur ceux au 1/10 000 et au 1/5000. Cette permanence est vérifiée par comparaison des Plans directeurs d'échelles différentes. En revanche, l'accroissement du nombre de segments corrélatif à l'échelle scalaire (on observe davantage de boyaux et de tranchées au 1/5000 qu'au $1 / 20000$ pour une même surface) induit sur la densité toponymique (Chasseau, 2017). La désignation des tranchées est généralement pérenne dans le temps. En effet, la comparaison des Plans directeurs éditées à des dates différentes entre 1915 et 1918 sur un même secteur témoigne non seulement de la densification, de la multiplication des positions, des changements structuraux des réseaux de défense mais aussi de la permanence des noms. Cette dernière concerne également les tranchées alliées prises à l'ennemi suite à une offensive.

\section{La toponymie du champ de bataille ne se résume pas aux noms de tranchées}

Conformément aux manuels militaires (GQGA, 1916; 1917), tous les éléments importants de la planimétrie ou du relief doivent recevoir des appellations correspondant soit à ceux de la carte d'État-major antérieure aux Plans directeurs, soit au cadastre napoléonien. Le cas échéant, les nouveaux noms déjà employés par les troupes et une désignation aléatoire sont proposés. Si certains noms de lieux et microtoponymes ruraux préexistants au conflit ont subsistés et ont été appropriés par les combattants - comme le Chemin des Dames, le Col du Linge, les forts de Verdun, le «Mort-Homme »- d'autres sont nés de la Grande Guerre et du jargon militaire. Ces " polémo-toponymes » hérités de la Grande Guerre, continuent à perpétuer la mémoire comme la « Cote 108 » à Berry-au-Bac, ou le « Bois Sabot » à Souain-Perthes-Lès-Hurlus.

Les bois et les pinèdes de la Champagne sèche sont référencés par des lettres, des numéros et des formes quand la morphologie du massif est caractéristique comme les « bois en T », « bois parallèles », « bois horizontal ", « bois en U », « bois en Y ", « bois Sabot ", « bois en Pioche ", " bois Carré », « bois du Boomerang ». Les reliefs participent également au choix des toponymes comme «la Main de Massiges » sur la Cote de Champagne dont le tracé digité rappelle la forme d'une main (illustration 7). Les " marsouins » (régiment d'infanterie de marine) désignent ce site dont chaque saillant séparé par un ravin, correspond à un doigt de la main avec d'ouest en est, « le Faux Pouce », «le Pouce », "l'Index", « le Médius » et "l'Annulaire». Dans le dispositif allemand, au nord du «Creux de l'Oreille », la «Chenille» prolongée par la " Tête de Vipère » illustre également l'utilisation des oronymes et des métaphores anatomiques dans la toponymie du champ de bataille. 
Illustration 7 - La « Main de Massiges »

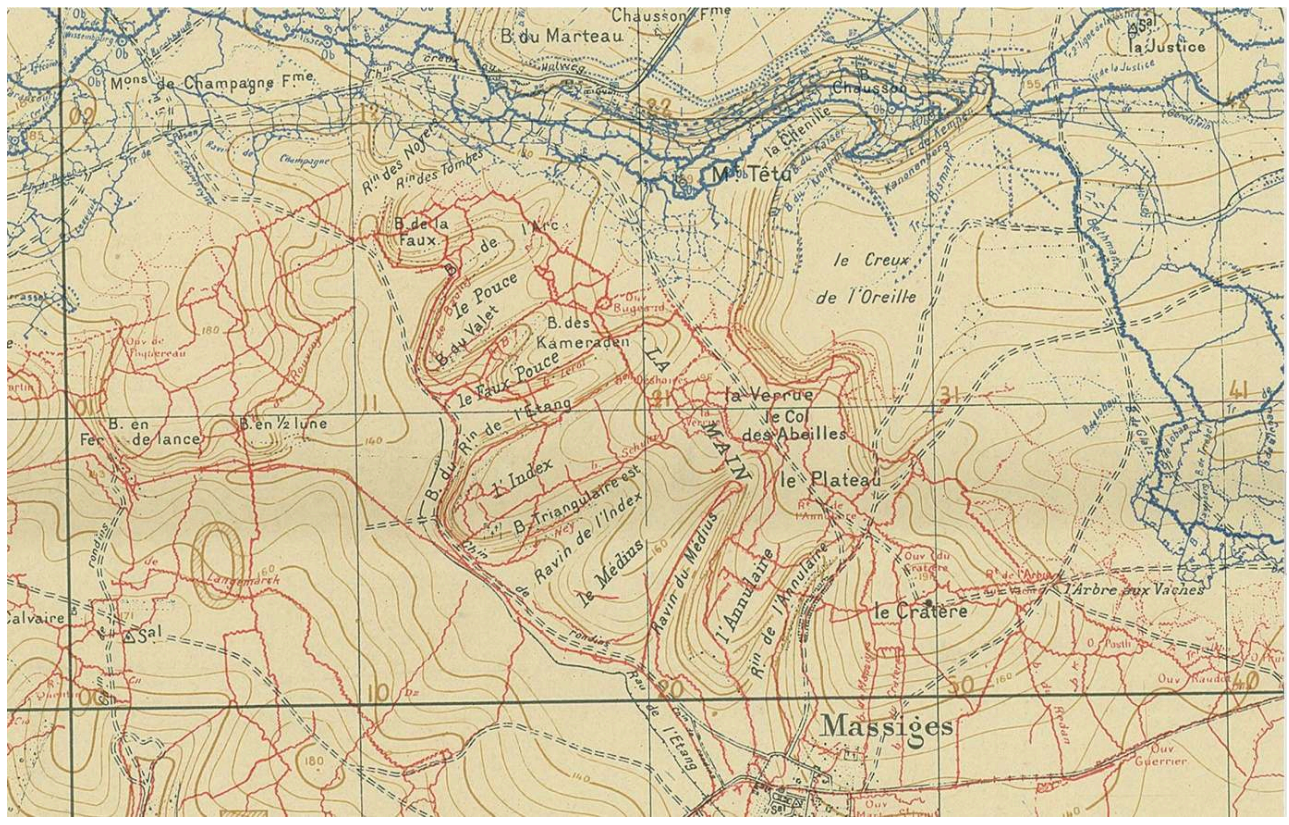

Source : extrait du Plan directeur au 1/20 000 de Cernay-en-Dormois du 19/09/1918, coll. personnelle.

Les polémo-toponymes font souvent référence aux altitudes et plus spécifiquement aux " cotes ». Représentées en noir et précédées du mot "Cote » sur les Plans directeurs, elles représentent les points hauts de la carte d'État-major au 1/80 000, dont l'altitude est corrigée (nouveau nivellement) par une cote en caractère droit et de couleur bistre. Le polémo-toponyme colporte ainsi une valeur erronée d'altitude dont la comparaison avec l'altitude réelle s'explique trop souvent, à tort dans l'opinion collective par ablation liée à la bombturbation. Cette dernière, avec la guerre des mines, participe néanmoins à de profonds bouleversements morphologiques (Weiss, 2015).

À l'image des «Cote 204 » à Château-Thierry (illustration 7), «Cote 304 » à Esnes-enArgonne, «Cote 108 » à Berry-au-Bac (Taborelli et al., 2017a), ou de la « Cote 295 » du Mort-Homme près de Verdun (illustration $8 \mathrm{~B}$ ), les «Cotes » véhiculent des lieux de combats acharnés, des sites profondément affectés par la Grande Guerre. Cette appropriation territoriale s'inscrit dans la mémoire collective des sociétés par des monuments, des mémoriaux et des nécropoles. Elle peut dépasser les frontières à l'image du village de "Vimy-Ridge » au Canada en mémoire à la crête de Vimy trop souvent représentée comme lieu d'affirmation d'indépendance au sein du Commonwealth (Martin, 2014). 

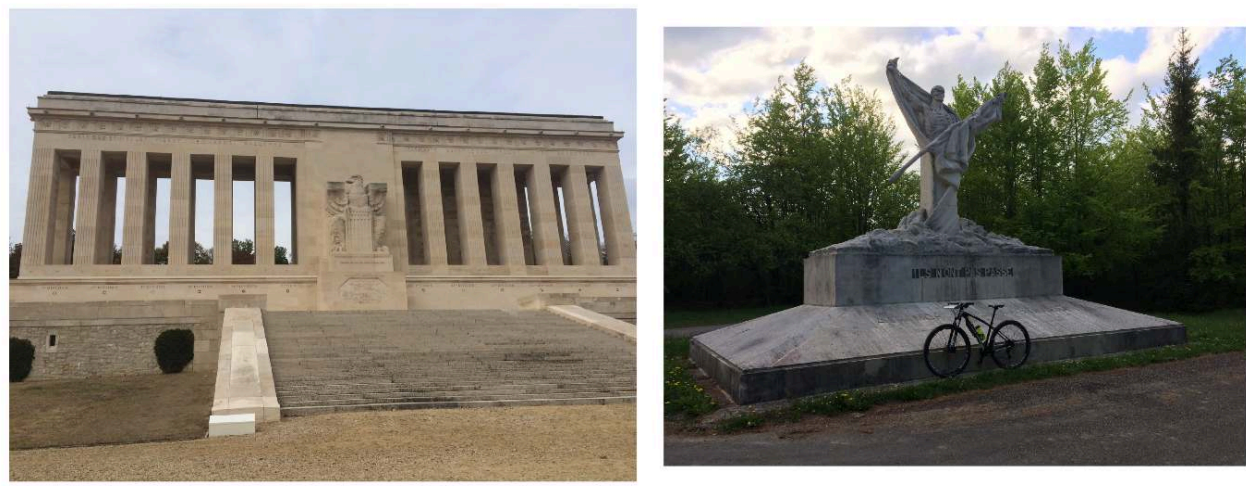

Gauche : A. Monument américain de la Cote 204 à Château Thierry - Droite : B. Monument de la Cote 295 ou « Le Squelette » de la 69ème Division d'Infanterie du Mort-Homme près de Verdun. Auteur : Alain Devos.

\section{Conclusion}

La toponymie est particulièrement utilisée durant la Grande Guerre, dans le cadre de la guerre de position pour permettre la géolocalisation des soldats dans le réseau de défense, dense et complexe. Elle accompagne l'appropriation du terrain si chèrement gagné par les combattants. L'approche spatiale sous SIG des noms de tranchées à partir des Plans directeurs de la Grande Guerre en Champagne et leur typologie apportent de nombreuses réponses sur leur densité, leur répartition, et leur détermination. Leur distribution bimodale (essentiellement des toponymes et des patronymes) cache une remarquable diversité culturelle dont l'organisation spatiale souligne une paternité régimentaire associée à un "quartier» de la zone de front. Cette appropriation du terrain par les combattants se manifeste par une répartition territoriale des noms de tranchées à l'échelle locale autour de thèmes culturels très diversifiés, renforçant l'esprit de corps et la valeur identitaire du groupe.

La toponymie du champ de bataille ne se résume pas qu'aux noms de tranchées mais utilise aussi les éléments importants de la planimétrie. Des sites sont taxés de nouveaux toponymes durant le conflit (polémo-toponymes). Ces derniers s'inscrivent aujourd'hui dans la mémoire collective et correspondent à des sites mémoriels de la Grande Guerre.

La valeur opérationnelle de la banque de données toponymique des noms de tranchées est vérifiée dans le cadre de l'archéologie préventive, de la valorisation patrimoniale, des recherches généalogiques (géolocalisation des morts pour la France), et du zonage des risques géotechniques (aléas cavités) et pyrotechniques. Elle s'exprime davantage avec les héritages morphologiques de la Grande guerre révélés par le lidar (Taborelli et al., 2017b ; De Matos-Machado et al., 2019).

Cette contribution inédite soulève de nombreux questionnements scientifiques (paternité, origine des désignations, permanence scalaire et temporelle) et mériterait une extension à l'ensemble de la zone du front ouest et une comparaison avec les Trench maps et les Stellungskarten pour y répondre. À partir de cette nouvelle banque de données toponymiques géoréférencées, une véritable analyse spatiale multivariée pourrait être menée sur les $750 \mathrm{~km}$ du front ouest. 
Weiss J.F., 2015. Les souterrains de la Première Guerre mondiale, du creusement au témoignage, Tome 2. Saint-Cyr-l'Ecole, Éd. AREESVS, 223 p.

\section{BIBLIOGRAPHIE}

Amat J-P, 1987. Guerre et milieux naturels : les forêts meurtries de l'Est de la France, 70 ans après Verdun. L'Espace géographique, vol. 16, n³, p. 217-233. DOI: https://doi.org/10.3406/spgeo. 1987.4253

Amat J.-P., 2015. Les forêts de la Grande Guerre : histoire, mémoire, patrimoine. Paris, Presses de l'Université Paris-Sorbonne, $548 \mathrm{p}$.

Brénot J., Saulière N., Léty C., et al., 2017. How much did the soldiers dig? A quantification of WW1 remains in Argonne, France. Geoarcheology, n 32, p. 534-548. DOI: https://doi.org/10.1002/gea. 21623

Cancès B., Chabert C., Taborelli P.,2018. Un effet de la bombturbation : la contamination des sols par les éléments traces métalliques. In Bergerat F. (dir.), 14-18, La Terre et le Feu. Géologie et géologues sur le front occidental. Co-édition AGBP - COFRHIGÉO - SGN, Mém. hors-série n¹0 de l'AGBP, p. 411-413.

Cao F., Jaunat J., Sturchio N., et al., 2019. Worldwide occurrence and origin of perchlorate ion in waters: A review. Science of the Total Environment, n 661, p. 737-749. DOI: https://doi.org/10.1016/ j.scitotenv.2019.01.107

Chasseaud P., 2017. Rats alley, Trench names of the Western Front 1914-1918. Briscombe Port Stroud, Gloucestershire, Éd. The History Press, 767 p.

Combaud A., Devos A., Chalumeau L., et al., 2016. Les Plans directeurs de la Grande Guerre : une nécessaire innovation cartographique. Revue internationale de Géomatique, vol. 26, $\mathrm{n}^{\circ} 3$, p. 363-393.DOI : https://doi.org/10.3166/rig.2016.00004

Danchin E., 2011. Destruction du patrimoine et figure du soldat allemand dans les cartes postals de la Grande Guerre. Amnis, Revue d'étude des sociétés et cultures contemporaines Europe-Amérique [En ligne], n 10. URL : http://journals.openedition.org/amnis/1371 - DOI: https://doi.org/10.4000/ amnis.1371

De Matos-Machado R., Amat J.-P., Arnaud-Fassetta G., Bétard F., 2016. Potentialités de l'outil LiDAR pour cartographier les vestiges de la Grande Guerre en milieu intraforestier (bois des Caures, forêt domaniale de Verdun, Meuse). ÉchoGéo [En ligne], n³8. URL : https:// journals.openedition.org/echogeo/14791

De Matos-Machado R., Toumazet J-P., Bergès J-C., et al., 2019. War landform mapping and classification on the Verdun battlefield (France) using airborne LiDAR and multivariate analysis. Earth Surface Processes and Landforms, vol. 44, n 7, p. 1430-1448. DOI: https://doi.org/10.1002/esp. 4586

Desfossés Y., Jacques A., Prilaux G., 2008. L'archéologie de la Grande Guerre. Rennes, Co-édition Ouest-France et Inrap, $127 \mathrm{p}$. 
Devos A., Taborelli P., Dodici M., et al., 2015. Rôle des conditions géographiques sur l'organisation spatiale des réseaux de défense de la Grande Guerre. Application à la Champagne (région de Reims). Physio-Géo, n 9, p. 157-174. URL : https://journals.openedition.org/physio-geo/4615

Devos A., Taborelli P., Damien T., et al., 2017. La Grande Guerre sur la Côte d'île-de-France, en Champagne crayeuse et en Argonne, Journées d'Etudes d'Automne 8-9 octobre 2016. Bulletin d'Informations Géologiques du Bassin de Paris, vol. 54, n 3, p. 3-19.

Devos A., Taborelli P., Laratte S., 2018a. Les paysages de guerre. In Bergerat F. (dir.), 14-18, La Terre et le Feu. Géologie et géologues sur le front occidental. Co-édition AGBP - COFRHIGÉO - SGN, Mém. hors-série ${ }^{\circ} 10$ de l'AGBP, p. 395-403.

Devos A., Taborelli P., Desfossés Y. 2018b. L'organisation spatiale du front : une question d'échelle ! In Bergerat F. (dir.), 14-18, La Terre et le Feu. Géologie et géologues sur le front occidental. Coédition AGBP - COFRHIGÉO - SGN, Mém. hors-série nº 10 de l'AGBP, p. 68-81.

Devos A., Bollot N., Laratte S., et al., 2019a. «Creutes » et réseaux de défenses dans les paysages de guerre - Exemple du Mont-Chatté (Hermonville, France). Revue de Géographie Historique [En ligne], $\mathrm{n}^{\circ}$ 14-15. Actes du colloque IMPACT 14-18, 20-21 septembre 2018, Reims. URL : http://rgh.univlorraine.fr/articles/view/116/

Devos A., Taborelli P., Laratte S., Lesjean F., 2019b. Un exemple de structure cellulaire de défense : la butte de Brimont. Apport des Plans directeurs et du Lidar (France). Revue de Géographie Historique [En ligne], n 14-15. Actes du colloque IMPACT 14-18, 20-21 septembre 2018, Reims. URL : http://rgh.univ-lorraine.fr/articles/view/115/

Un_exemple_de_structure_cellulaire_de_defense_la_Butte_de_Brimont_Apport_des_plans_directeurs_et_du_Lidar_France

Gheyle W., Saey T., Van Hollebeeke Y., et al., 2016. Historical Aerial Photography and Multireceiver EMI Soil Sensing, Complementing Techniques for the Study of a Great War Conflict Landscape. Archaeological prospection, vol. 23, n 3, p. 149-164. DOI: https://doi.org/10.1002/arp. 1534

GQGA - Grand Quartier Général des Armées - État-Major, 1916. Instruction sur les Plans directeurs. Paris, Imprimerie nationale, $45 \mathrm{p}$.

GQGA - Grand Quartier Général des Armées - État-Major, 1917. Instruction sur les Plans directeurs et les cartes et plans spéciaux : cette instruction abroge l'instruction sur les Plans directeurs du 14 février 1916. Paris, Imprimerie Nationale, $37 \mathrm{p}$.

Hesse R., 2014. Geomorphological traces of conflict in high-resolution elevation models. Applied geography, n 46, p. 11-20. DOI: https://doi.org/10.1016/j.apgeog.2013.10.004

Hubé D., 2016. Sur les traces d'un secret enfoui, enquête sur l'héritage toxique de la Grande Guerre. Paris, Éd. Michalon, 285 p.

Hubé D., 2018. 14-18, Tremblements de guerre, les géologues au cœur de l'Histoire. Orléans, Éd. BRGM, $192 \mathrm{p}$.

Hupy J.P., Schaetzl R., 2006. Introduction “bombturbation", a singular type of soil disturbance and mixing. Soil Science, vol. 171, $\mathrm{n}^{\circ} 11$, p. 823-836. DOI: https://doi.org/10.1097/01.ss. 0000228053.08087 .19

Ministère des Armées, 1915. Journal des Marches et des Opérations, $247^{\circledR}$ Régiment d'Infanterie. Du 4 août 1914-1er octobre 1915. Service Historique de la Défense. SHD GR 26N727/6.

Longnon A., 1891. Dictionnaire topographique du département de la Marne comprenant les noms de lieu anciens et modernes, publication par ordre du ministre de l'Instruction publique. s/dir. du Comité des travaux historiques. Imprimeries nationales, $380 \mathrm{p}$. 
Martin J., 2014. Un siècle d'oubli. Les Canadiens et la première Guerre mondiale (1914-2014). Outremont, Éd. Athéna, Coll. Histoire militaire, 235 p.

Masson-Loodts I., 2014. Paysages en bataille, les séquelles environnementales de la Grande Guerre. Bruxelles, Éd. Nevicata, $102 \mathrm{p}$.

Parent G.H., 1991. Etudes écologiques et chorologiques sur la flore lorraine. Note 10. Les plantes obsidionales de la Lorraine. Bulletin de la Société d'Histoire Naturelle de la Moselle, $\mathrm{n}^{\circ}$ 45, p. 45-110.

Sainéan L., 1915. L'argot des tranchées d'après les lettres des poilus et des journaux du front. Éd. E. De Boccard, $163 \mathrm{p}$.

Stichelbaut B., 2011. The first thirty kilometers of the western front 1914-1918: An aerial archeological approach with historical remote sensing data. Archeological Prospection, vol. $18, \mathrm{n}^{\circ} 1$, p. 57-66. DOI: https://doi.org/10.1002/arp.397

Taborelli P., Devos A., Dodici M., et al., 2016. Apport des Plans directeurs sur la compréhension de l'organisation spatiale du front durant la Grande Guerre. Application à l'Argonne. Revue de Géographie Historique [En ligne], n 8. URL: http://rgh.univ-lorraine.fr/articles/view/66/

Taborelli P., Devos A., Laratte S., et al., 2017a. Apport des Plans directeurs et de l'outil lidar aéroporté pour la caractérisation des impacts morphologiques de la Grande Guerre - exemple de la cote 108 (Berry-au-Bac - France). Géomorphologie : Relief, Processus, Environnement, vol. 23, n 2, p. 155-169. DOI: https://doi.org/10.4000/geomorphologie.11711

Taborelli P., Devos A., Laratte S., et al., 2017b. Typologie et organisation spatiale des « polémoformes » de la Grande Guerre révélées par l'outil Lidar et les plans directeur. Application à la Champagne et à l'Argonne. Revue de Géographie Historique [En ligne], $\mathrm{n}^{\circ}$ 10. URL : http://rgh.univlorraine.fr/articles/view/88/

Taborelli P., Devos A., Laratte S., Brénot J., 2018. The geomorphological control on the western front of the Great War in Champagne and Argonne (France). Scientia Militaria South African Journal of Military Studies, vol. 46, n 1, p. 149-161. DOI: https://doi.org/10.5787/46-1-1229

Tamine M., 2012. Le hêtre dans la toponymie des Ardennes. Nouvelle revue d'onomastique, $\mathrm{n}^{\circ} 54$, p. 37-74.

Vernier F., 2006. Les plantes obsidionales, ces plantes qui suivent les troupes. In Martin P., Simiz S. (dir.), L'empreinte de la guerre : de la Grèce classique à la Tchétchénie, Panazol, Éd. Lavauzelle, p. 533-540.

Vilatte R., 1925. Les conditions géographiques de la guerre, étude de géographie militaire sur le front français de 1914 à 1918. Paris, Éd. Payot, 325 p.

Wearn J-A., Budden A-P., Veniard S-C, Richardson D., 2017. The flora of the Somme battlefield: A botanical perspective on a post-conflict landscape. First War Studies, $\mathrm{n}^{\circ} 8$, p. 63-77. DOI: https:// doi.org/10.1080/19475020.2017.1348959

\section{RÉSUMÉS}

Cette contribution porte sur la toponymie des champs de bataille et plus particulièrement sur les noms des tranchées et des boyaux de la Grande Guerre. Elle vise à les caractériser, et à comprendre leur organisation spatiale. La méthode de travail repose sur un inventaire, une typologie et une cartographie sous SIG, des noms de tranchées et de boyaux, réalisé à partir des Plans directeurs des Groupes de Canevas de Tirs des Armées au 1/20 000 de 1918, sur un linéaire de $115 \mathrm{~km}$ de front en Champagne. Les résultats valorisés par une analyse graphique et 
cartographique sur 2500 noms répartis sur $13000 \mathrm{~km}$ de réseaux de défense, montrent une diversité étonnante. Leur désignation aléatoire conventionnelle cache en réalité une organisation spatiale en groupes de segments de tranchées et de boyaux ou "quartiers", d'origine régimentaire et non divisionnaire. Les toponymes et les noms de tranchées constituent une représentation socio-culturelle de la zone de front et s'inscrivent aujourd'hui dans le patrimoine mémoriel et collectif des sociétés. Ils soulèvent de nombreux questionnements scientifiques (paternité, origine des désignations, permanence scalaire et temporelle) et mériteraient une extension à l'ensemble de la zone du front ouest, ainsi qu'une analyse spatiale approfondie.

The toponymy of battlefield and more particularly the trench names of the Great War remains little known without spatial analysis or statistics. The study of trench maps reveals the western front is made of a dense and complex defense network structured according to the geomorphological conditions. To improve the operational aspect of trench maps, the soldiers increased the names, designations and numbers associated with nameboards. The typological study of the 2,500 trench names of Champagne, on $13,000 \mathrm{~km}$ of linear for $115 \mathrm{~km}$ of front, and their spatial organization from a database under GIS, allows us to better understand the naming logic of these places. Their astonishing diversity and their conventional random designation actually hide a spatial organization into groups of fire trench segments and communication trench or "quarters", of regimental and non-divisional origin. This organisation around cultural themes guarantees the movement of troops and liaison officers in the defense network. The toponyms and trench names constitute a socio-cultural representation of the front area and are today part of the memorial and collective heritage of societies. Trench names raise a lot of scientific questions (authorship, origin of designations, scalar and temporal permanence) and deserve a spatial analysis of the entire western front area.

\section{INDEX}

Keywords : trench names, Great War, Champagne, spatial organisation, GIS

Mots-clés : nom de tranchée, Grande Guerre, Champagne, organisation spatiale, SIG

Thèmes : Sur le Champ - Sur le Terrain

\section{AUTEURS}

\section{ALAIN DEVOS}

Alain Devos, alain.devos@univ-reims.fr, est professeur des Universités à l'Université de Reims Champagne-Ardenne et membre de l'UMR GEGENAA. Il a récemment publié :

- Devos A., Bollot N., Laratte S., Taborelli P., Fronteau G., 2019. «Creutes » et réseaux de défenses dans les paysages de guerre - Exemple du Mont-Chatté (Hermonville, France). Revue de Géographie Historique, $\mathrm{n}^{\circ}$ 14-15. [En ligne] http://rgh.univ-lorraine.fr/articles/view/116/ Creutes_et_reseaux_de_defenses_dans_les_paysages_de_guerre_exemple_du_Mont_Chatte_Hermonville_France - Devos A., Taborelli P., Larattte S., Lesjean F., 2019. Un exemple de structure cellulaire de défense : la butte de Brimont. Apport des Plans directeurs et du Lidar (France). Revue de Géographie Historique, ${ }^{\circ}$ 14-15. [En ligne] http://rgh.univ-lorraine.fr/articles/view/115/ Un_exemple_de_structure_cellulaire_de_defense_la_Butte_de_Brimont_Apport_des_plans_directeurs_et_du_Lidar_France - Devos A., Taborelli P., Damien T., et al., 2017. La Grande Guerre sur la Cote d'île-de-France, en Champagne crayeuse et en Argonne-Journées d'étude d'Automne. Bulletin de l'Association des Géologues du Bassin de Paris (AGBP), vol. 54, n 3, p. 3-19. 


\section{PIERRE TABORELLI}

Pierre Taborelli, pierre.taborelli@total-quadran.com, est docteur en géographie, Chef de projet à TOTAL-QUADRAN, Agence Grand-Est/Hauts de France, Châlons-en-Champagne. Il a récemment publié :

- Taborelli P., Devos A., Laratte S., Brenot J., 2018. The geomorphological control on the western front of the Great War in Champagne and Argonne (France). Scientia Militaria South African Journal Of Military Studies, vol. 46, $\mathrm{n}^{\circ}$ 1, p. 149-161.

- TaborelliP., DevosA., LaratteS., et al., 2017. Apport des Plans directeurs et de l'outil lidar aéroporté pour la caractérisation des impacts morphologiques de la Grande Guerre - exemple de la côte 108 (Berry-au-Bac, France). Géomorphologie, vol. 23, n² 2, p. 155-169.

- Brenot J., Saulière N., Léty C., et al., 2017. How much did the soldiers dig? A quantification of WW1 remains in Argonne, France. Geoarchaelogy, vol. 32, n 5, p. 1-15.

\section{ROBIN PERARNAU}

Robin Perarnau, robin.perarnau@univ-reims.fr, est ingénieur d'études à l'Université de Reims Champagne-Ardenne et membre de l'UMR GEGENAA. Il a récemment publié :

- Brenot J., Desfossés Y., Perarnau R., et al., à paraître. The practice of Trench warfare: the training camps in the Aube (Champagne region, France), 1914-1919. Proceedings of the $13^{\text {th }}$ International Conference on Military Geosciences, Padova (Italy), 24-28 June 2019.

- Coulaud A., Mariette M. et Perarnau R., 2019. L'empreinte américaine durant la Grande Guerre Persistance des vestiges américains en France. Revue de Géographie Historique, n 14-15. [En ligne] http://rgh.univ-lorraine.fr/articles/view/113/

L_empreinte_americaine_durant_la_Grande_Guerre_persistances_des_vestiges_americains_en_France_1917_1919

- Coulaud A. et Perarnau R. en collaboration avec Charrière G. et Mariette M., 2018. La baraque militaire dans les hôpitaux américains de la Grande Guerre. Stratégies économiques et choix socio-culturels. Archéopages, nº 46, p. 80-85. 